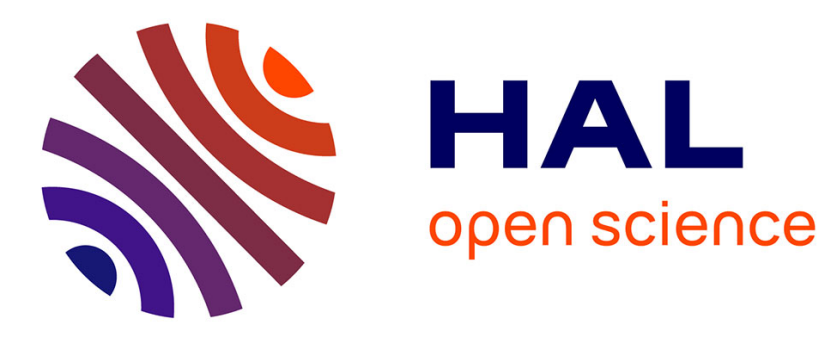

\title{
Oversolubility in the microvicinity of solid-solution interfaces
}

Isabelle Bergonzi, Lionel Mercury, Patrick Simon, Frederic Jamme, Kirill Shmulovich

\section{- To cite this version:}

Isabelle Bergonzi, Lionel Mercury, Patrick Simon, Frederic Jamme, Kirill Shmulovich. Oversolubility in the microvicinity of solid-solution interfaces. Physical Chemistry Chemical Physics, 2016, 18, pp.14874-14885. 10.1039/C5CP08012F . insu-01308355

\section{HAL Id: insu-01308355 \\ https://hal-insu.archives-ouvertes.fr/insu-01308355}

Submitted on 27 Apr 2016

HAL is a multi-disciplinary open access archive for the deposit and dissemination of scientific research documents, whether they are published or not. The documents may come from teaching and research institutions in France or abroad, or from public or private research centers.
L'archive ouverte pluridisciplinaire HAL, est destinée au dépôt et à la diffusion de documents scientifiques de niveau recherche, publiés ou non, émanant des établissements d'enseignement et de recherche français ou étrangers, des laboratoires publics ou privés.

\section{(ㅇ)(1) $\$$}

Distributed under a Creative Commons Attribution - NonCommercial - NoDerivatives| 4.0 

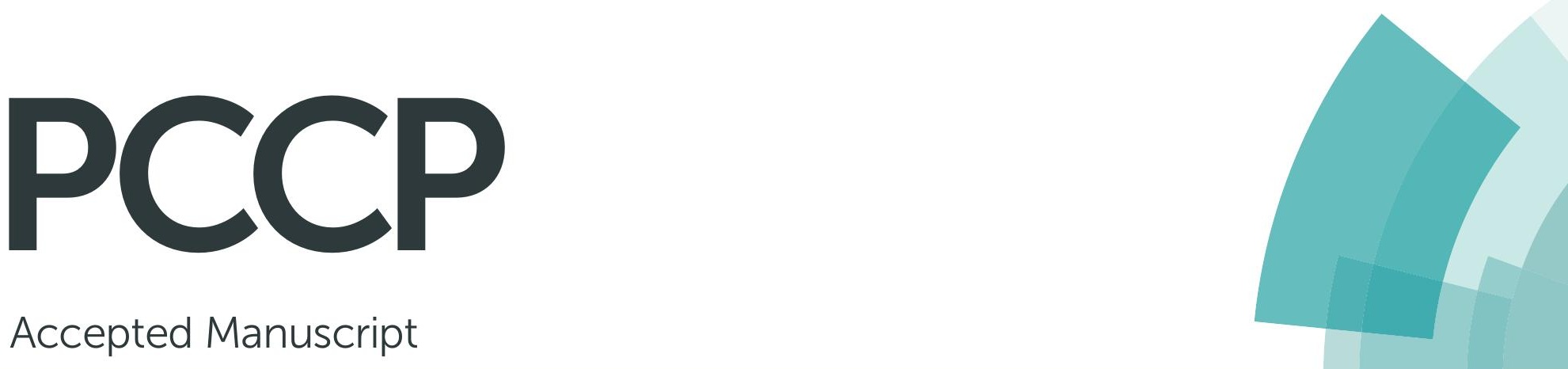

\section{Accepted Manuscript}

This article can be cited before page numbers have been issued, to do this please use: I. Bergonzi, L. Mercury, P. Simon, F. Jamme and K. Shmulovich, Phys. Chem. Chem. Phys., 2016, DOI:

\subsection{9/C5CP08012F.}

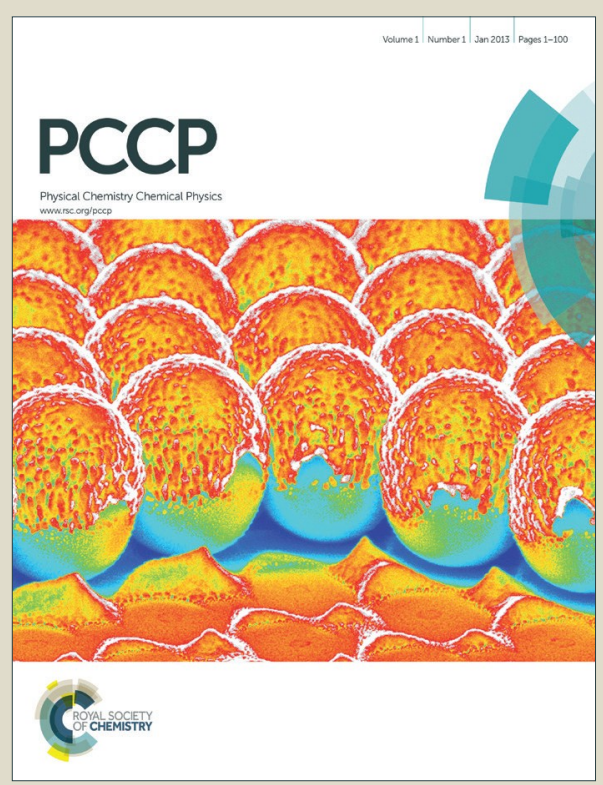

This is an Accepted Manuscript, which has been through the Royal Society of Chemistry peer review process and has been accepted for publication.

Accepted Manuscripts are published online shortly after acceptance, before technical editing, formatting and proof reading. Using this free service, authors can make their results available to the community, in citable form, before we publish the edited article. We will replace this Accepted Manuscript with the edited and formatted Advance Article as soon as it is available.

You can find more information about Accepted Manuscripts in the Information for Authors.

Please note that technical editing may introduce minor changes to the text and/or graphics, which may alter content. The journal's standard Terms \& Conditions and the Ethical guidelines still apply. In no event shall the Royal Society of Chemistry be held responsible for any errors or omissions in this Accepted Manuscript or any consequences arising from the use of any information it contains. 


\section{Oversolubility in the microvicinity of solid-solution interfaces}

Isabelle Bergonzi ${ }^{1}$, Lionel Mercury ${ }^{1, *}$, Patrick Simon $^{2}$, Frédéric Jamme $^{3}$, Kirill Shmulovich ${ }^{4}$

${ }^{1}$ Institut des Sciences de la Terre d'Orléans, UMR 7327 Université d'Orléans/ CNRS/ BRGM, 45071 Orléans cedex, France.

${ }^{2}$ CNRS, CEMHTI UPR3079, Université d'Orléans, CS 90055, 45071 Orleans Cedex 2.

${ }^{3}$ Synchrotron SOLEIL, L’Orme des Merisiers, Saint Aubin, BP 489, 91192 Gif-sur-Yvette, France.

4 Institute of Experimental Mineralogy, Russian Academy of Science, 142432 Chernogolovka, Russia.

* Corresponding author: 1ionel.mercury@univ-orleans.fr

Keywords: Liquid water; pores, channels, cells; Interfacial effect; Vibrational spectroscopy; Gibbs free energy.

Running head: Interfacial oversolubility 


\begin{abstract}
Water-solid interactions at macroscopic level (beyond ten of nanometers) are often viewed as the coexistence of two bulk phases with a sharp interface in many areas spanning from biology to (geo)chemistry and various technological fields (membranes, microfluidics, coatings, etc.). Here we present experimental evidence indicating that such a view may be a significant oversimplification. High-resolution infrared and Raman experiments were performed in a $60 \times 20 \mu \mathrm{m}^{2}$ quartz cavity, synthetically created and initially filled with demineralized water. The IR mapping $\left(3 \times 3 \mu \mathrm{m}^{2}\right.$ beam size $)$ performed with the SOLEIL synchrotron radiation source displays two important features: (i) the presence of a dangling free-OH component, a signature of hydrophobic inner walls; (ii) a shift of the $\mathrm{OH}$-stretching band which essentially makes the $3200 \mathrm{~cm}^{-1}$ sub-band to predominate over the usual main component around $3400 \mathrm{~cm}^{-1}$. Raman maps confirmed these signatures (though less marked than IR's) and afford a refined spatial distribution of this interfacial signal. This spatial resolution, statistically treated, results in a puzzling image of a 1-3 $\mu \mathrm{m}$ thick marked-liquid layer along the entire liquid-solid interface. The common view is then challenged by these strong evidences that a $\mu \mathrm{m}$-thick layer analogous to an interphase forms at the solid-liquid interface. The thermodynamic counterpart of the vibrational shifts amounts to around +1 $\mathrm{kJ} / \mathrm{mol}$ at the interface with a rapid decreasing signature towards the cavity centre, meaning that vicinal water may form a reactive layer, micrometer thick, expected to have an elevated melting point, a depression of the boiling temperature, and enhanced solvent properties.
\end{abstract}




\section{INTRODUCTION}

Liquid water imbibes almost every open space in natural or synthetic materials on Earth. Its very low viscosity, high surface tension, high dipole moment, high dielectric constant, all contribute to the ability of liquid water to easily penetrate almost everywhere and to coexist with any material. In the same time, the water solvent properties lead the containers to react with the occluded liquid according to the laws of chemical affinity. Water-solid interactions (e.g., in rock pores) can be generally described based on the properties of bulk phases, especially for liquid water, except for the nanometric distances from solid surfaces ${ }^{1-5}$. However, there also exist multiple evidences at the macroscopic scale that do not fit this scheme, especially observations about the pore-size dependent solubility of minerals which become sensitive at the micrometer (and not the nm) scale ${ }^{6-12}$.

It was also found that the behavior of water trapped in technological or synthetic cavities changes with the (macroscopic) size of the cavity. A recent review ${ }^{5}$ highlights many atypical measurements recorded on water trapped in channels of 50-500 nm dimensions. Atypical behaviors were even recorded inside $1 \mu \mathrm{m}$ (or more) cavities, when water interacts with silanized hydrophobic surfaces ${ }^{13}$. Authors showed, by NMR measurements, that the water molecules in extended nanospaces (at least up to size between $750 \mathrm{~nm}$ and $1.5 \mu \mathrm{m}$ ) are coupled in the direction perpendicular to the surface.

Biological organisms and organics also show a large variation in their behavior when in contact with thick water films (up to thousands of molecules), demonstrating the complex interplay between the size of the intracellular space, the quantity and the nature of the local $\operatorname{organics}^{14-16}$

This paper reports on high-resolution experiments designed to characterize the thermodynamic state of liquid water in micro-cavities, in situations as closest as possible to natural interactions between aqueous solution and minerals, at the one-pore scale. Vibrational 
properties of water are carefully mapped over the entire cavity with a $2.5 \times 2.5 \mu \mathrm{m}$ step size (then smaller than the beam size) and linked to the effect of the interfacial state of confined water. After conversion with an adequate partition function ${ }^{17}$, the Gibbs free energy of water appears to be dependent on the distance to the confining surface up to the micrometer length scale. The consequences of this behavior which can be expected in terms of inpores/channels/cells solubility are eventually discussed.

\section{MATERIALS AND METHODS}

\subsection{Material: Synthetic Fluid Inclusion}

The micro-cavities in a solid crystal (here, quartz) hosting an occluded liquid (here, water) are prepared using an hydrothermal procedure to fabricate synthetic fluid inclusions (SFIs) ${ }^{18}$. The hydrothermal synthesis was performed in internally-heated pressure vessels (IHPV, or "gas bomb") ${ }^{19}$ with quartz prisms, firstly thermally cracked to open internal micro-cavities. The cracked prisms were then put in Pt capsules together with triple distilled water $(100 \mu l)$ and amorphous silica $(10 \mathrm{mg})$. The synthesis was carried out at $610 \mathrm{MPa}$ and nominally $458^{\circ} \mathrm{C}$ during 41 days, meaning the trapped liquid water has got a density of around $0.92 \mathrm{~g} / \mathrm{cm}^{3}$. At these conditions, the liquid, initially available in the capsule, was trapped in the micro-cavities (SFIs) while amorphous silica turned into quartz, sealing the cracks and creating closed cavities, logically termed SFIs. The final step is to return back to room conditions at which the inclusions-bearing quartz fragment can be recovered, prepared and observed. At room conditions, the trapped liquid coexists with a bubble of saturated water vapor (all other gases are degassed from the solution in the preparatory stage), and is made of the initially in-capsule liquid (pure water, presently), enriched by a small amount of silica acid dissolved during the synthesis. As the quartz solubility at hydrothermal conditions largely exceeds the room's ones, it is expected a quartz reprecipitation onto the cavity walls during and after the 
cooling/depressurizing process, promoting both smooth inner walls, and an equilibrium shape of the inclusion.

After a years-long waiting time to favour equilibrium conditions, one fluid inclusion was selected in one inclusions-bearing quartz fragment (Fig. 1). The SFI is approximately $60 \mu \mathrm{m}$ x $20 \mu \mathrm{m}$ in size and displayed a clear shape of a negative quartz crystal, indicating equilibrium growth conditions. To get the accurate 3D geometry of the inclusion at micrometric resolution, X-ray micro-tomography measurements were performed on the ID19 beamline at ESRF (Fig. 1). The thickness of the inclusion is $5 \mu \mathrm{m}$ and is hardly changing over most of the inclusion, except only close to the two extremities where it appears to become slightly thinner.
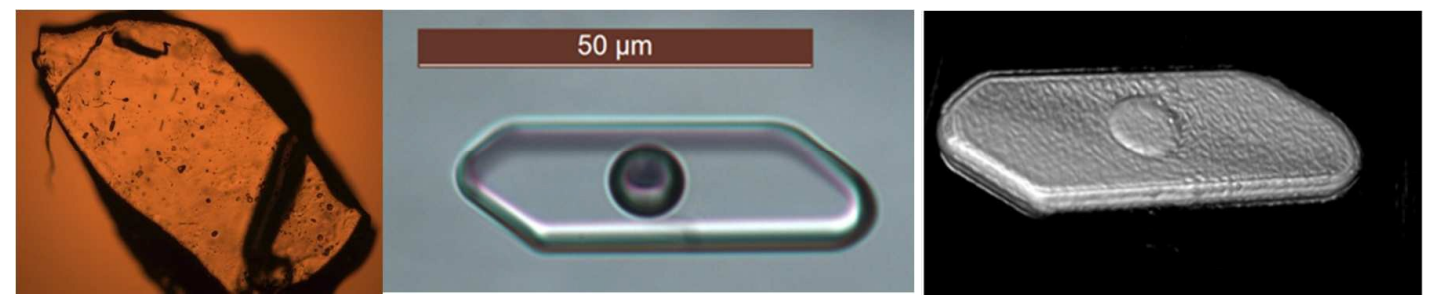

Figure 1. From left to right: quartz fragment containing fluid inclusions (black dots); micrographic and tomographic images of the selected inclusion.

\subsection{Experimental setup}

\subsubsection{X-ray tomography}

The sample was imaged on the 3-D microtomography ( $\mu$-CT) unit developed on the beamline ID 19 at the ESRF. The system utilizes a large monochromatic parallel X-ray beam $(20 \mathrm{keV}$, pink beam mode) and a 2-D area detector. The specimen to be imaged is mounted on a translation/rotation stage allowing precise alignment in the beam. The main surface of the sample $(5 \mathrm{~mm} \times 5 \mathrm{~mm})$ is set perpendicular to the incident beam. To compile a 3-D image set for a material, a series of 1200 radiographic images ( $0.5 \mathrm{~s}$ per image) are recorded at different angular positions from $0^{\circ}$ to $180^{\circ}$. After conversion to light by a fluorescent scintillator 
screen, the radiographic images are digitized using a FReLoN camera. The objective was a 60X Olympus, and the distance from the sample to the scintillator was $10 \mathrm{~mm}$.

A 3-D filtered back-projection algorithm, PyHST2, developed at ESRF, is then used to reconstruct a 3-D image of the specimen from the series of 2-D projections. For the operating conditions and optical setup employed in this study, it was possible to acquire an image with a voxel (linear) dimension of $0.12 \mu \mathrm{m}$, and so the spatial resolution is around $0.5 \mu \mathrm{m}$.

\subsubsection{Infrared measurements}

Micro-FTIR experiments were performed at the SOLEIL Synchrotron (Gif sur Yvette, France) at the SMIS beamline, equipped with a Contin $\mu$ um XL microscope coupled to a Nicolet 5700 FT-IR spectrometer (Thermo Fisher Scientific, USA). The microscope comprises a liquid nitrogen cooled mercury cadmium telluride (MCT) detector $(50 \mathrm{~mm})$ with a $32 \mathrm{X}$ infinity corrected Schwarzschild type objective $(\mathrm{NA}=0.65)$ and a matching $32 \mathrm{X}$ condenser, working in dual confocal mode ${ }^{21}$.

Owing to the quality of the radiation source, a double path single masking aperture size of $3 \mathrm{x}$ $3 \mu \mathrm{m}$ has been reached with a good $\mathrm{S} / \mathrm{N}$ ratio, and the inclusion mapped with a step size of 2.5 $\mu \mathrm{m} \times 2.5 \mu \mathrm{m}$. Each spectrum was recorded in the $2500-4500 \mathrm{~cm}^{-1}$ spectral range, with a spectral resolution of $8 \mathrm{~cm}^{-1}$ with 200 scans per spectrum and no mathematical correction. The infrared absorption coefficient is defined by $A=-\log \left(\frac{I}{I_{0}}\right)$, where $I_{0}$ is the transmitted intensity of the quartz crystal and $I$ the transmitted intensity of quartz and liquid-filled inclusion.

We strongly outline the beam size, quite unusual in IR studies, associated with a good enough $\mathrm{S} / \mathrm{N}$ ratio to ascertain that the signals can be quantitatively treated. Also, the same maps were acquired at one year distance to ensure that we were recording equilibrium-based signals. Another noteworthy point is the dual confocal aperturing optical arrangement ${ }^{21}$ which creates the best conditions to reach a spatial resolution at the diffraction limit. In the work presented, 
in order to obtain the best performance at $3 \mu \mathrm{m}\left(3400 \mathrm{~cm}^{-1}\right)$, a dual mask aperture has been used in transmission mode geometry coupled with the high brightness of the synchrotron source (SMIS beamline, SOLEIL synchrotron, Gif sur Yvette, France).

\subsubsection{Raman measurements}

Raman spectra were obtained in the backscattering geometry with a Renishaw Invia Reflex Raman spectrometer equipped with a DM 2500 Leica microscope $(100 \times$ objective, NA = 0.85 ) in confocal mode, a 600 grooves/mm grating, edge filter, and a $633 \mathrm{~nm}$ excitation line of a He-Ne laser (power 10mW).

The spectra were acquired over a rectangular zone of 2160 points with a step distance of 1 $\mu \mathrm{m}$. The exposure time for each point was $20 \mathrm{~s}$, leading to a total acquisition time of 12 hours. Data treatment and Principal Component Analysis (PCA) were performed with Renishaw Wire 3.4 software.

\section{RESULTS}

\subsection{Tomographic observations}

At the beamline resolution, the inclusion appears to be a 5-6 $\mu \mathrm{m}$ thick elongated hexagon, with sharp borders. In particular, the inclusions walls, either on the sides or at the top/bottom of the cavity, display almost planar shapes without sensitive curvature in the resolution scope. This special geometry is therefore attractive by exposing a lot of liquid-solid interfacial surface area at reasonably constant optical pathway.

\subsection{Infrared results}

\subsubsection{Inclusion-sized maps}

The $\mathrm{OH}$ stretching band is the only one that can be recorded in quartz due to the silica absorption below $2000 \mathrm{~cm}^{-1}$. Fortunately, it is known as a very sensitive probe of the 
hydrogen bonds (H-bonds hereafter) networking ${ }^{22}$, and can be used to convert vibrational energy into thermodynamic properties ${ }^{17}$.

Two "raw" (without any correction) absorbance maps (Fig. 2) drawn at $3400 \mathrm{~cm}^{-1}$ (the principal vibrational mode of bulk water) and $3700 \mathrm{~cm}^{-1}$ (normally not expressed in bulk water) illustrate that the $\mathrm{OH}$ stretching band is substantially modified when moving from the centre of the fluid inclusion to the cavity walls $(2.5 \mu \mathrm{m}$ step size). The former "bulk" frequency maximally absorbs when far away from the solid-liquid (walls) and liquid-air (bubble) interfaces, while the latter absorption grows close to the liquid/solid and liquid/air interfaces. Additionally, close to the interface, the absorbance is systematically very low (due to the reduced amount of probed water) and particularly at high frequencies (between 0.009 abs. u. to 0.217 abs. u. at $3700 \mathrm{~cm}^{-1}$ ).

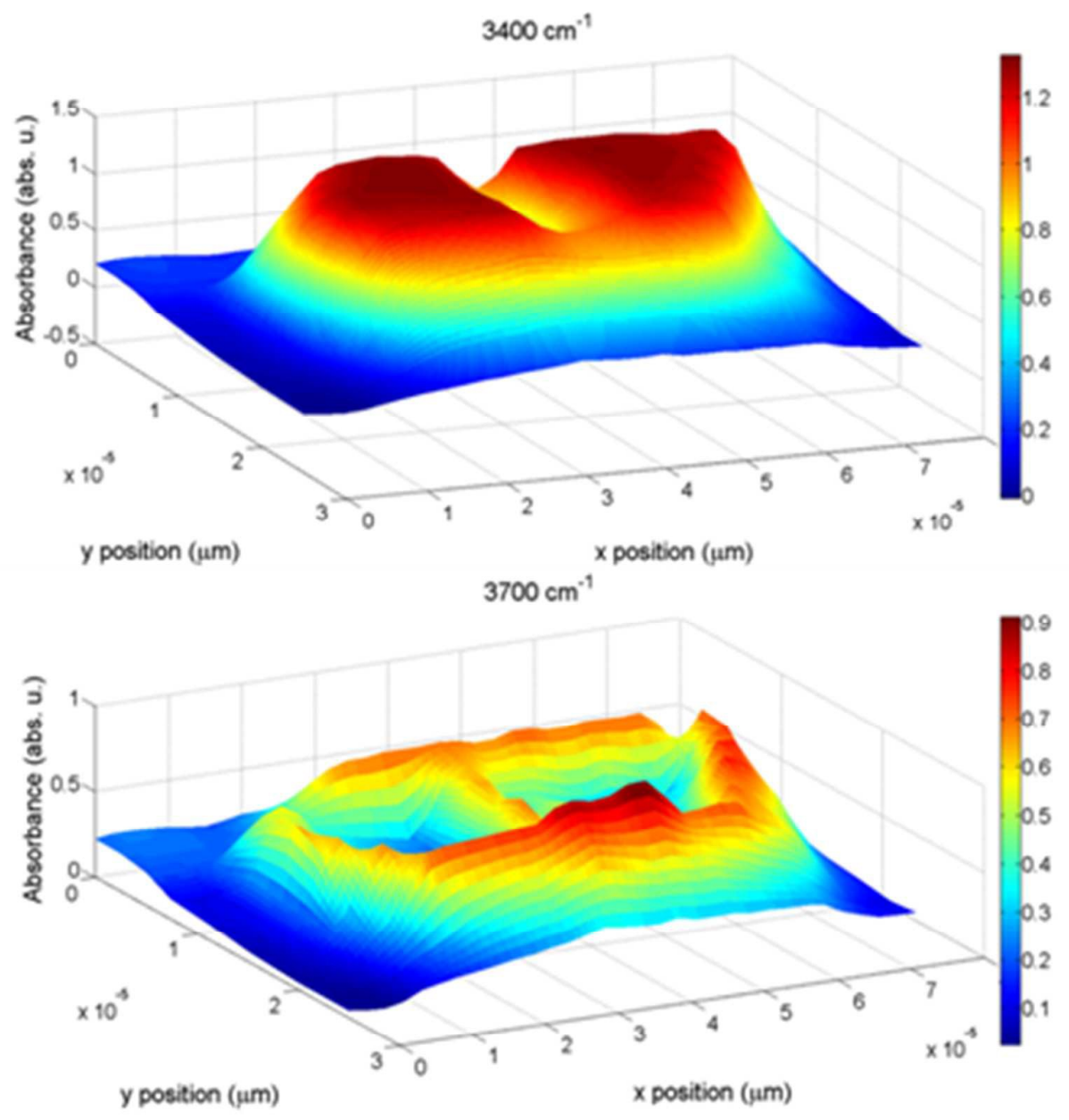


Figure 2. Infrared raw maps of the fluid inclusion at two specific wavenumbers.

\subsubsection{Detailed analysis of the $\mathrm{OH}$ stretching band}

The baseline correction of the $\mathrm{OH}$-stretching band was performed by the Matlab function "Backcorr" using a truncated cost function ${ }^{23}$, and the corrected spectra are closely studied in terms of the band main frequency, absorbance and width, mapped as a function of the beam location in the inclusion (Fig. 3).
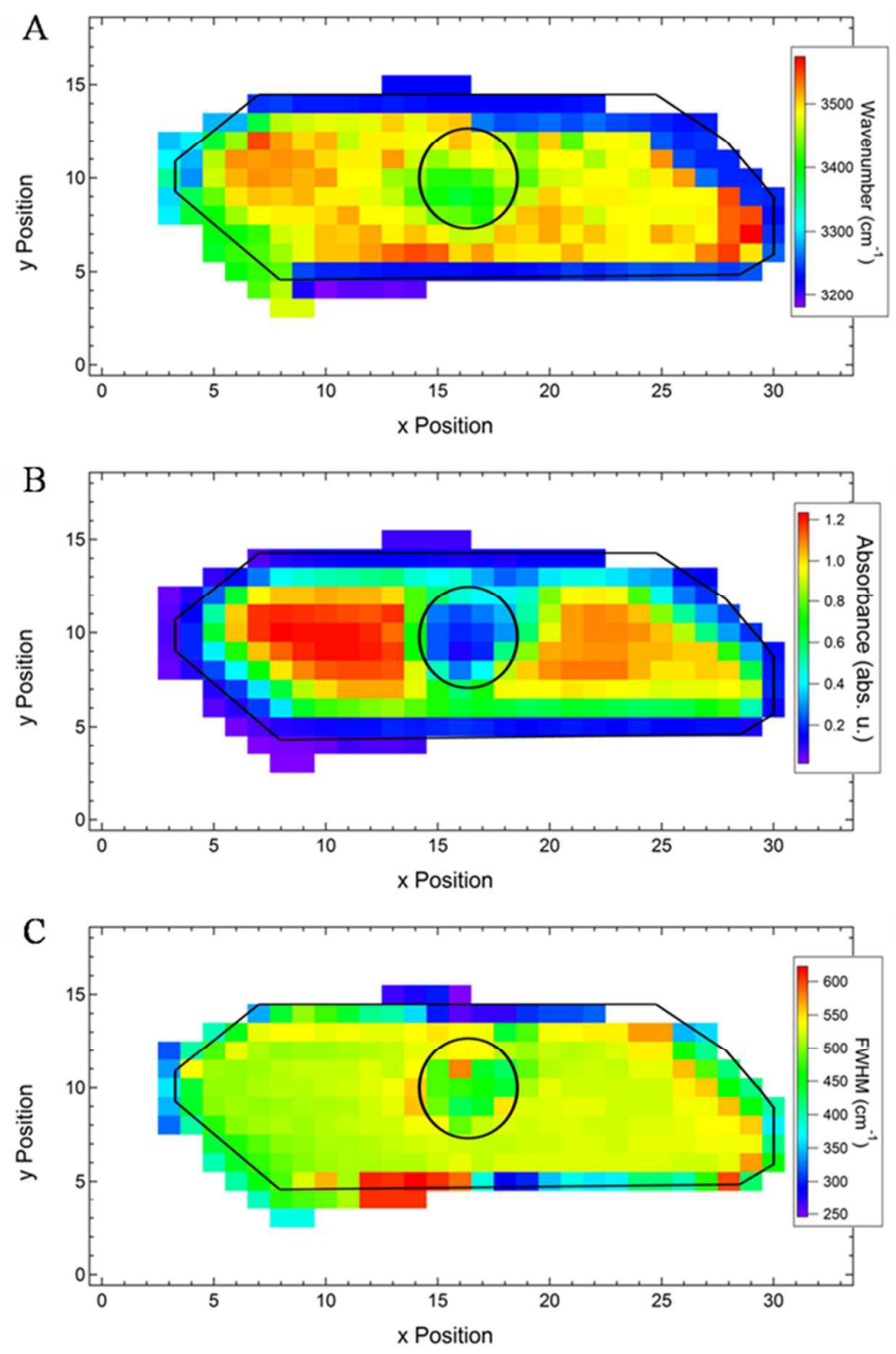

Figure 3. Maps of the main features of the $\mathrm{OH}$ stretching band of $\mathrm{H}_{2} \mathrm{O}$ as a function of the beam localization (numbered arithmetically in $\mathrm{x}$ and $\mathrm{y}$ ) in the inclusion. A: Main frequency 
(in $\mathrm{cm}^{-1}$ ) of the band. B: Maximal absorbance (in abs. units). C: Full width at half maximum (FWHM, in $\left.\mathrm{cm}^{-1}\right)$.

The main frequency of the band (Fig. 3A) is a probe of the dominant spectral (and then energetic) mode in water. In the centre of the inclusion, it is quite constant at $3490 \mathrm{~cm}^{-1}$, shifted to higher frequencies than the bulk value $\mathrm{e}^{17,22,24}$. Close to the solid/liquid and liquid/air interfaces, it becomes down-shifted to $3250 \mathrm{~cm}^{-1}$, in agreement with previous studies ${ }^{25-26}$. The down-shifting is generally interpreted as an increasing connectivity and strengthening of the water H-bonding network, resulting in the main frequency coming closer to the $\mathrm{OH}$-stretching band of ice. Both interfaces show the down-shift but the effect is weaker with the liquid/vapor interface than with the liquid/solid one.

The maximal absorbance of the band (Fig. 3B) is a probe of the local water amount. It is higher in the centre of the inclusion and decreases progressively toward the interfaces, most probably related to a thinning of the inclusion.

The full width at half maximum (FWHM) of the band (Fig. 3C) is a probe of the spectral range (and, hence, the energetic range) of local water. Approaching the liquid/solid and liquid/vapor interfaces tends to decrease the FWHM of the $\mathrm{H}_{2} \mathrm{O}$ stretching band with respect to the molecules in the centre of the inclusion. At the same time, the "interface-marked" water band becomes larger than the one of the bulk water.

After this first analysis, the baseline-corrected spectra taken along nine cross sections in the inclusion (Fig. 4) were studied. It appears that the band shape is always significantly modified as a function of the distance from the surface. Farther from the interfaces, the bands are more or less similar to the bulk ones, except for the largest FWHM (about $+35-40 \%$ ). Closer to the interfaces, the spectra display two main shapes. Along A, B and I cross sections, the FWHM is about $15-20 \%$ higher than in the bulk water spectrum and the vibrational band has a stronger lower frequency component. Moreover, an increasing absorbance at the end of the 
band is visible, due to a large undulation evident in raw spectra. Along C, D, E, F, G and H cross sections, the FWHM is about $65-80 \%$ higher than in the bulk water spectrum. The $\mathrm{OH}$ stretching band again has a stronger lower frequency component, but also exhibits a strong shoulder at higher frequencies located at $3665-3752 \mathrm{~cm}^{-1}$. Even discarding this latter shoulder, the FWHM along the C, D, E, F, G and $\mathrm{H}$ cross sections are narrower at the edges than in the centre. Qualitatively, this trend is consistent with a restricted energetic range of water close to the edges, which is itself completely compatible with the up-shift of the main frequency.

The most surprising result is the rise of a new mode at high frequencies $\left(3665-3752 \mathrm{~cm}^{-1}\right)$, visible from place to place along the quartz cavity walls, and along the air-water bubble interface. Actually, this frequency is well known as the IR absorption of free-OH or dangling$\mathrm{OH}$ modes, widely observed at the water/hydrophobic interfaces (including water-air interfaces), particularly by SFG (Sum-Frequency Generation ${ }^{28}$ ), SFVS (Sum-Frequency Vibrational Spectroscopy ${ }^{29-31}$ ), and with TIR-VSFS (total internal reflection vibrational sum frequency spectroscopy ${ }^{32}$ ). These spectroscopic tools resolve the first nanometers from the surface, and at this scale the $\mathrm{OH}$ stretching band notably displays one sharp peak at $3700 \mathrm{~cm}^{-}$ ${ }^{1}$, assigned to the dangling- $\mathrm{OH}$ at the interface ${ }^{33}$, indistinctly from interfacial $\mathrm{H}_{2} \mathrm{O}$ molecules or surface silanols. Accordingly, the shoulder observed at $\left(3665-3752 \mathrm{~cm}^{-1}\right)$ is assigned to the same dangling $\mathrm{OH}$ sub-band, despite a beam size largely beyond the distances scrutinized by the surface spectroscopies. While the air-water interfaces are notoriously hydrophobic, the additional surprise was to observe this dangling mode along the inner walls of quartz cavity, meaning them to be hydrophobic. In other words, according to this measurement, they experience a low connectivity with the adjacent water molecules, the water-quartz interface is poorly connected by H-bonds, and the terminal water molecules have to organize themselves without contact or "continuity" with the first layers of the solid wall surfaces. 


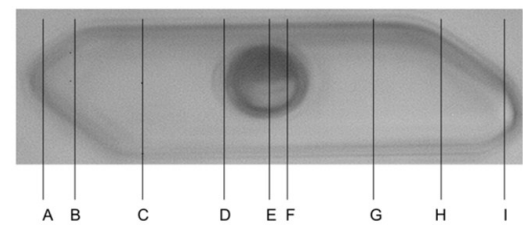

A
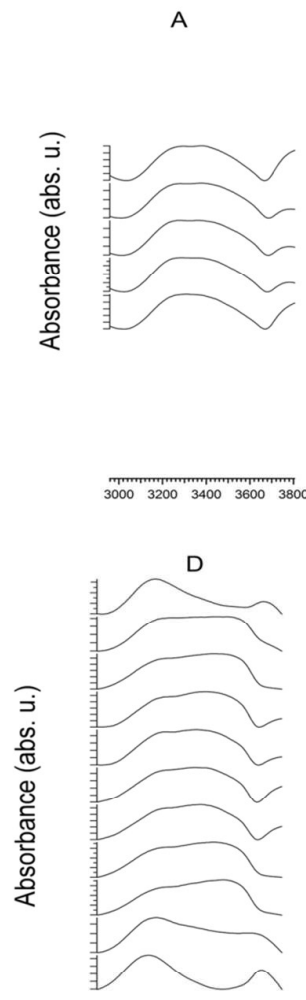

$3000 \quad 320034003600 \quad 3800$

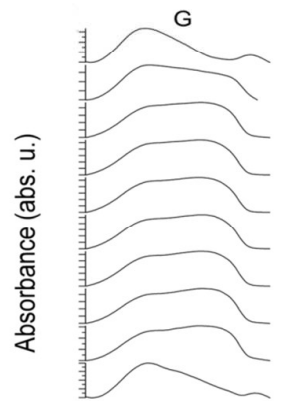

$3000 \quad 3200 \quad 3400 \quad 3600 \quad 3800$
B
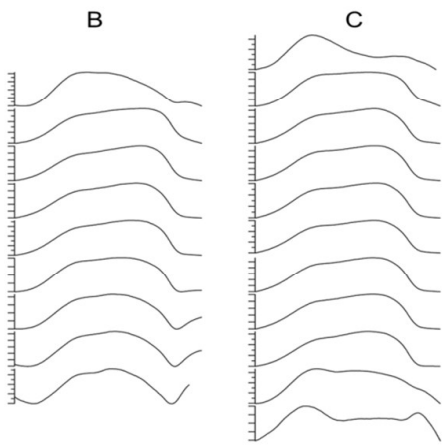

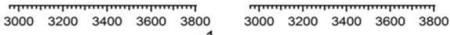
Wavenumber $\left(\mathrm{cm}^{-1}\right)$
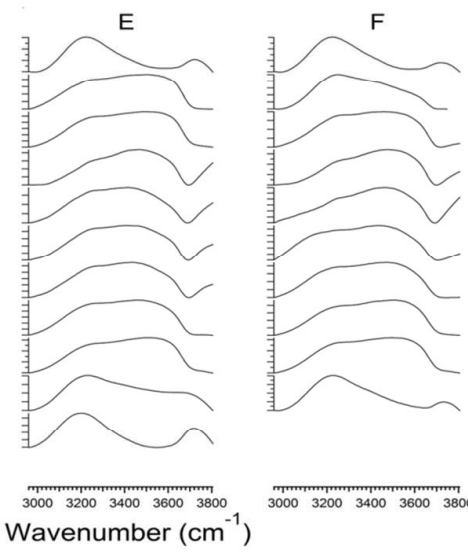

$\mathrm{H}$
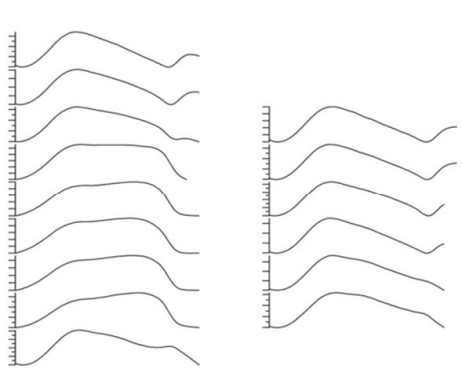

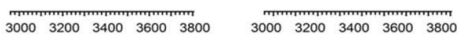

Wavenumber $\left(\mathrm{cm}^{-1}\right)$

Figure 4. Nine series of spectra along nine cross sections of the inclusion. Their positions are indicated on the photograph of the inclusion. 
Despite the classic assumption of a systematic hydroxylation of water-contacting silica, this hydrophobic nature of quartz inner walls is compatible with the high $(P, T)$ conditions of the hydrothermal synthesis $\left(610 \mathrm{MPa}, 458^{\circ} \mathrm{C}\right)$. Actually, they have promoted the formation of high density of siloxane bridges, the hydrolysis of which at ambient conditions is very unlikely, due to its high activation barrier ${ }^{39-40}$. Additionally, several recent Molecular Dynamics simulations demonstrated that a siloxane-dominated surface is hydrophobic ${ }^{32-38}$. A further evidence about the high quality quartz growth process is the negative quartz crystal shape displayed by many inclusions in the quartz fragment, including the inclusion under study.

Before stepping forward, one question arises about the discontinuity of this IR signature of interfaces along the inner walls. In particular, a large undulation of the baseline is frequently recorded along the liquid-solid and air-liquid interfaces, and may hide, due to the broadness of the signal, the sharp dangling band. This large undulation of the baseline slightly distorts some of the interfaces-located spectra, specifically in the $3600-3700 \mathrm{~cm}^{-1}$ spectral range while the rest of the $\mathrm{OH}$-stretching band area appears almost not affected. This undulation is interpreted as a Mie scattering effect, related to the size and shape of the interface, as observed elsewhere ${ }^{27}$. A possibly roundly shape of the walls, not visible in the tomographic image, accompanied with the $5 \mu \mathrm{m}$ or less depth of the interface, makes the dimensions of the walls commensurate with the IR wavelengths. Moreover, the same undulation is especially visible close to the bubble (cross sections D, E and F in Fig. 4.), at which the spherical geometry drastically promotes the Mie effect.

To ensure that this explanation is correct, we performed a Raman study, not prone to Mie scattering, reported below in section 3.3. 


\subsubsection{Decomposition of the $\mathrm{OH}$ stretching band}

Decomposing the $\mathrm{OH}-$ stretching band into three Gaussian sub-components enables us to refine the interpretation of the state of water $^{24,26,41-42}$, but it is also a prerequisite for the thermodynamic interpretation of the IR data ${ }^{17}$. Actually, each Gaussian component can be assigned to water molecules with a given coordination number. The lower frequency Gaussian is assigned to the molecules tetrahedrally coordinated in a strong H-bonding network (Network Water, NW). The higher frequency Gaussian component can be ascribed to the molecules with lower coordination numbers (Multimer Water, MW). The Gaussian component centered between the previous two can be characterized by a coordination number close to three (Intermediate Water, IW). The spectra collected from the cavity were processed as described elsewhere ${ }^{17}$ to obtain the molecular fractions of each type of water. For the spectra collected close to interfaces, a fourth component was added to take the dangling-OH mode into account when present (Fig. 5, Table 1).
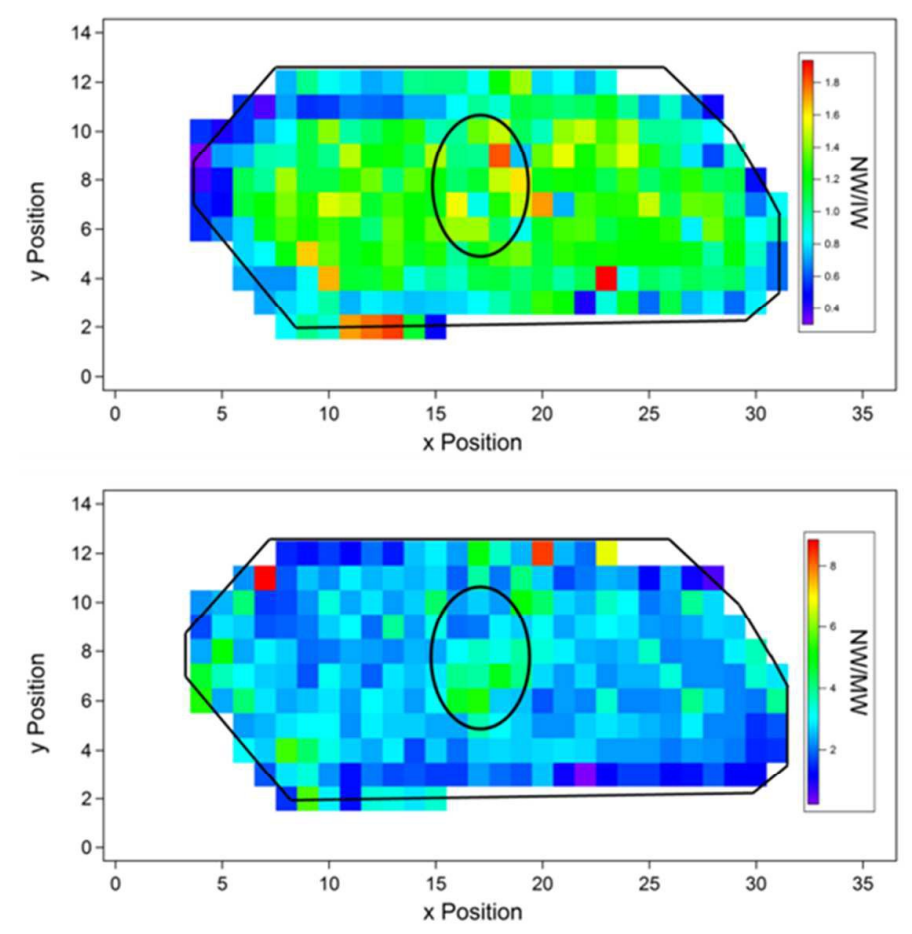

Figure 5. Maps of the NW/IW ratio (top) and NW/MW ratio (bottom). Certain spectra could not be decomposed safely, so they are not included (see Figure 3 by comparison). 
In the center of the inclusion, the NW/IW ratio is quite constant, oscillating from 1.2 to 1.4. Interestingly, the bulk values (unitary ratio) are not retrieved anywhere in the inclusion which means that the IR signal from water is perturbed everywhere in this large micrometric cavity. Closer to the interfaces the IW population becomes dominant over the NW population (usually dominant in the bulk), meaning that the molecular H-bonding network is affected by a decreasing connectivity. This finding is quite consistent with the emergence of the dangling mode to conclude that the quartz water interface is a low-connected (or hydrophobic) interface.

At the same time, the significant fraction of NW is well illustrated by the visual dominance of the $3200 \mathrm{~cm}^{-1}$ peak over the 3400 one (Fig. 4), demonstrating that the molecular water network is still highly interconnected. For comparison, let's recall that SFVS on water/OTScovered silica surface recorded similar features which were accounted for by the rigidity of the solid wall forcing the interfacial $\mathrm{H}_{2} \mathrm{O}$ molecules to form a more ordered molecular network while disconnected from the $\operatorname{solid}^{30,33}$. This explanation is also supported by molecular dynamics simulations ${ }^{36,43-44}$.

Table 1: Molecular fractions of three types of $\mathrm{H}_{2} \mathrm{O}$ molecules in the inclusion, calculated from the decomposition of the $\mathrm{OH}$-stretching band.

\begin{tabular}{c|c|c|c|c|c|c|}
\multicolumn{4}{c|}{ Liquid/solid interface } & \multicolumn{2}{c}{ Centre of the inclusion } \\
\hline \hline \multirow{2}{*}{ Mean fraction } & NW & IW & MW & NW & IW & MW \\
\cline { 2 - 7 } & 0.366 & 0.459 & 0.173 & 0.446 & 0.383 & 0.169 \\
\hline Maximal fraction & 0.542 & 0.705 & 0.587 & 0.532 & 0.595 & 0.234 \\
\hline Minimal fraction & 0.125 & 0.246 & 0.03 & 0.326 & 0.204 & 0.013 \\
\hline S.D. & 0.066 & 0.083 & 0.078 & 0.039 & 0.047 & 0.032 \\
\hline Bulk values $^{17}$ & & & & 0.587 & 0.307 & 0.106
\end{tabular}

\subsection{Raman spectroscopy}

Considering the surprising results obtained by micro-IR mapping, a complementary Raman study was performed. Actually, as water molecules have a very isotropic strong polarisability 
and a very directional H-bonding, the two spectroscopies are very sensitive to water but in a different way. Also, the size of the observation zone (focus point) is significantly different between the two techniques: close to $1 \mu \mathrm{m}^{2}$ for Raman, it is around one order of magnitude larger for IR. In terms of spectral range, and despite IR and Raman look at the same vibration mode, the absolute wavelength of the used electromagnetic waves is also very different: around $3000 \mathrm{~cm}^{-1}$ for IR, it is around $12800 \mathrm{~cm}^{-1}\left(15797 \mathrm{~cm}^{-1}\right.$, wavenumber of the He-Ne laser, minus $3000 \mathrm{~cm}^{-1}$ for Stokes shift) for Raman. Therefore, the two techniques operates at working electromagnetic frequencies separated by a factor of four, meaning any artifact effect should be noticeably different between IR and Raman.

Raman maps of the $\mathrm{OH}$ stretching band were recorded over the entire surface of the inclusion, taken at the optical plane (Fig. 6). First of all, the Raman maps did not show any baseline undulations, in close agreement to our hypothesis about the Mie scattering effect (laser wavelength is $633 \mathrm{~nm}$, the Stokes $\mathrm{OH}$ stretching spectral range stands then near $800 \mathrm{~nm}$ ). On the spectral side, the band intensity decreases close to both liquid/solid and vapor/liquid interfaces, as expected and already observed with IR spectroscopy. A close observation of the band shapes (Fig. 6, very representative) shows that the intensity of the peak centered near $3200 \mathrm{~cm}^{-1}$ catches up with the intensity of the peak at $3400 \mathrm{~cm}^{-1}$ when approaching the interface, in close agreement with the IR spectral evolution. However, this increasing contribution of the lower frequency component is less pronounced in the Raman spectrum and does not result in a dominance of this peak over the other. Also, the dangling mode is less visible, but appears clearly inside the liquid-solid interfacial layer and disappears in the center of the inclusion. This weaker spectral signature of the interface is consistent with the lower sensitivity of Raman spectroscopy to the changes of molecular environment. As a result, the two types of vibrational signals have a very satisfactory agreement. 


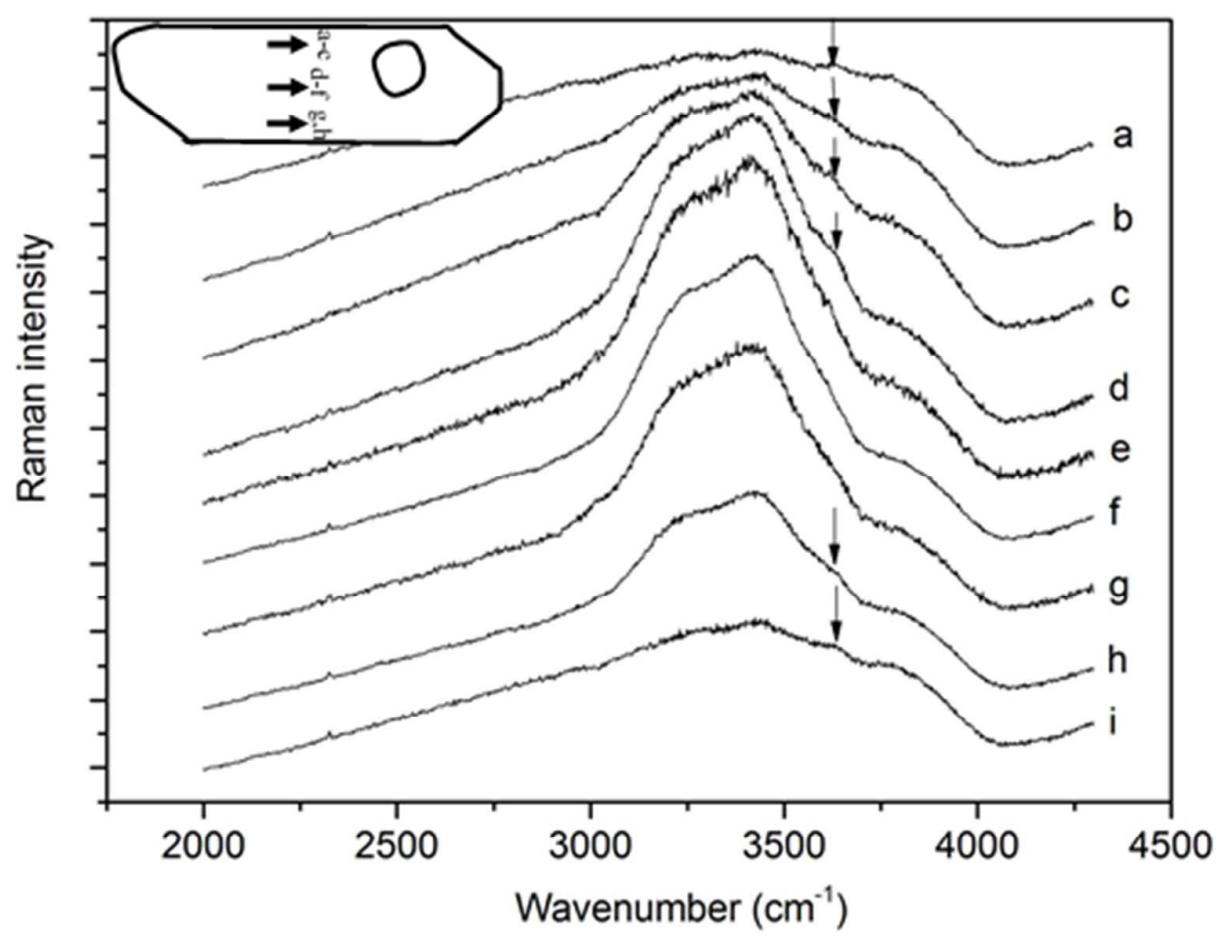

Figure 6. Raman $\mathrm{OH}$ stretching band of water at three characteristic locations in the inclusion. (a-c) Close to the top liquid/solid interface. (d-f) In the median part of the inclusion, far away any interface. $(\mathrm{g}, \mathrm{h})$ Close to the bottom liquid/solid interface.

We further performed principal component analysis (PCA) of the raw Raman spectra in the range 2000-4300 $\mathrm{cm}^{-1}$. PCA is a multivariate analysis adapted to analyze the intrinsic structure of the data, reducing the dimensionality of the data set by finding an appropriate set of coordinates, the principal components (PC). Variation of the PC scores of the PC1 as a function of the laser location in the inclusion (Fig. 7) demonstrates a significant change from the centre to the walls. Interestingly, while the PC1 definitely displays a $\mu$ m-thick layer distinct from the solid host and liquid guest, the PC2 only shows the transition from solid to liquid, and then a regular signal all inside the inclusion, without individualizing any thick interfacial layer. 


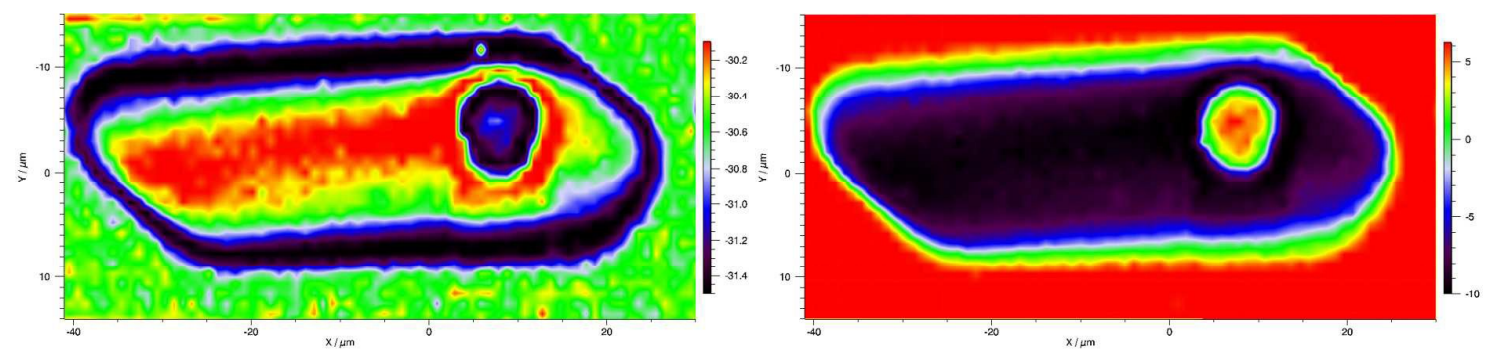

Figure 7. Score maps for PC1 (left) and PC2 (right) as a function of the laser beam location in the inclusion.

The PC scores provide an excellent cartography of the vicinal effect along the different interfaces. The regularity of the scores along the solid walls is a strong indication that the effect previously detected discontinuously by IR is a real and continuous interfacial effect. The scope can also be evaluated, from 0.5 to $3 \mu \mathrm{m}$, clearly thinner at the extremities: there is a probable influence of the wall shape/size to propagate the interfacial effect. Also, the watersolid and water-air interfaces are clearly marked and appear, at first sight, of the same intensity. For PC1, the most interesting aspect is the relative intensity variation from the thick layer close to the interface (black), in which the signal is constant, to the bulk water area (red), amounting to a significant $7 \%$ of the whole intensity.

The absence of any layering signal from PC2 is also interesting, demonstrating that the layer which is detected only by PC1, cannot be referred to a global "pollution" of the whole signal due (for instance) to optical aberration issues (see also next section).

The spectral features of the PCA components cannot be unambiguously interpreted, a usual limitation of the physical interpretation of the spectral-like features (the so-called loadings) given by PCA. Here, the shape of the loadings (Fig. 6) cannot be assigned to a change of the O-H bands alone: a broad luminescence background contributes to the loadings shape and precludes this univocal assignment. Then, we cannot discuss quantitatively the spectral side of this statistical analysis. Only the qualitative trend concerning the $3200 \mathrm{~cm}^{-1}$ and $3400 \mathrm{~cm}^{-1}$ 
peaks mentioned above can be ascertained, as well as the presence of a dangling sub-band along the solid-liquid interface.

\subsection{Optical aberration issues}

One could argue that the present results are related to optical distortion as, at the edges of the inclusion, the quartz-water interface may be curved (Mie scattering effect), and so refraction may arise when the light enters from an optically dense medium (quartz) into a less dense medium (water or air bubble). However, two complementary arguments can be proposed to support our current interpretation.

The first one is based on the similar geometrical extent of the thick interface effect obtained from IR and Raman techniques, while they operate at very different absolute frequency. Actually, they differ on three points:

- Physics and resulting optical conditions. IR is a transmission measurement, with similar numerical apertures on the arrival and departure sides of the sample. Raman is a light scattering experiment, with a very limited aperture for excitation, due to the limited laser beam diameter, and a larger one on the scattered light. These two very different geometries cannot be expected to induce the same aberrations.

- Focus point. As a direct consequence of the previous point, and as already mentioned, the size of the observation zone is very different between the two techniques.

- Distinct spectral range for IR and Raman. The optical effects of each technique are connected to a very different absolute wavelength of the used electromagnetic waves, as already mentioned, a four times gap. It is not reasonable to think that the effect of optical aberration might occur in the same manner at so different frequencies. If it was the case, such effect must be operating only at one of these two working frequencies, or, if operating at both, it must induce geometrical ghosts noticeably different between IR 
with and Raman. And besides this, in IR, the response should be affected by rapid changes of real and imaginary parts of the optical index due to $\mathrm{OH}$ bond vibration, while it is definitely not the case for Raman.

The second argument is about the PCA analysis on Raman map which provides two component scores, one with the thick interface figure, the other without it, as already mentioned (Fig. 7). Any optical (geometrical, refraction, etc...) aberration will induce identical effects on all PCA scores. If any optical aberration would take place, it must appear in all the PCA scores which are composed of slightly different spectral responses (the loadings) on the whole image. It is not possible to have a different aberration figure on different PCAs since they correspond to the same measurement.

As a conclusion, and despite a possible small (not visible at $500 \mathrm{~nm}$ resolution) curvature of the sides of the inclusion, possibly playing a minor role through the optical pathways of the probing lights, the convergent measurements reported above, do express a physico-chemical reality.

\section{INTERFACIAL THERMODYNAMICS}

The findings from vibrational spectroscopy can be expressed in thermodynamic terms of Gibbs free energy owing to an IR-to-thermodynamics conversion based on a partition function approach recently developed ${ }^{17}$. It relies on summing up the frequencies of all interand intra-molecular modes of $\mathrm{H}_{2} \mathrm{O}$ vibrations to deduce the Gibbs free energy. In the present case, due to the frequency cut-off from the quartz container, no other modes than the intramolecular stretching can be recorded. Therefore, the IR-to-thermodynamics conversion is first performed assuming that all the other modes, except for the intra-molecular stretching, are constant and equal to the bulk values (Fig. 8). As detailed below, a second calculation is 
carried out with both the stretching and the connectivity band (inter-molecular mode) changing, the bending and the libration again assumed to be constant.
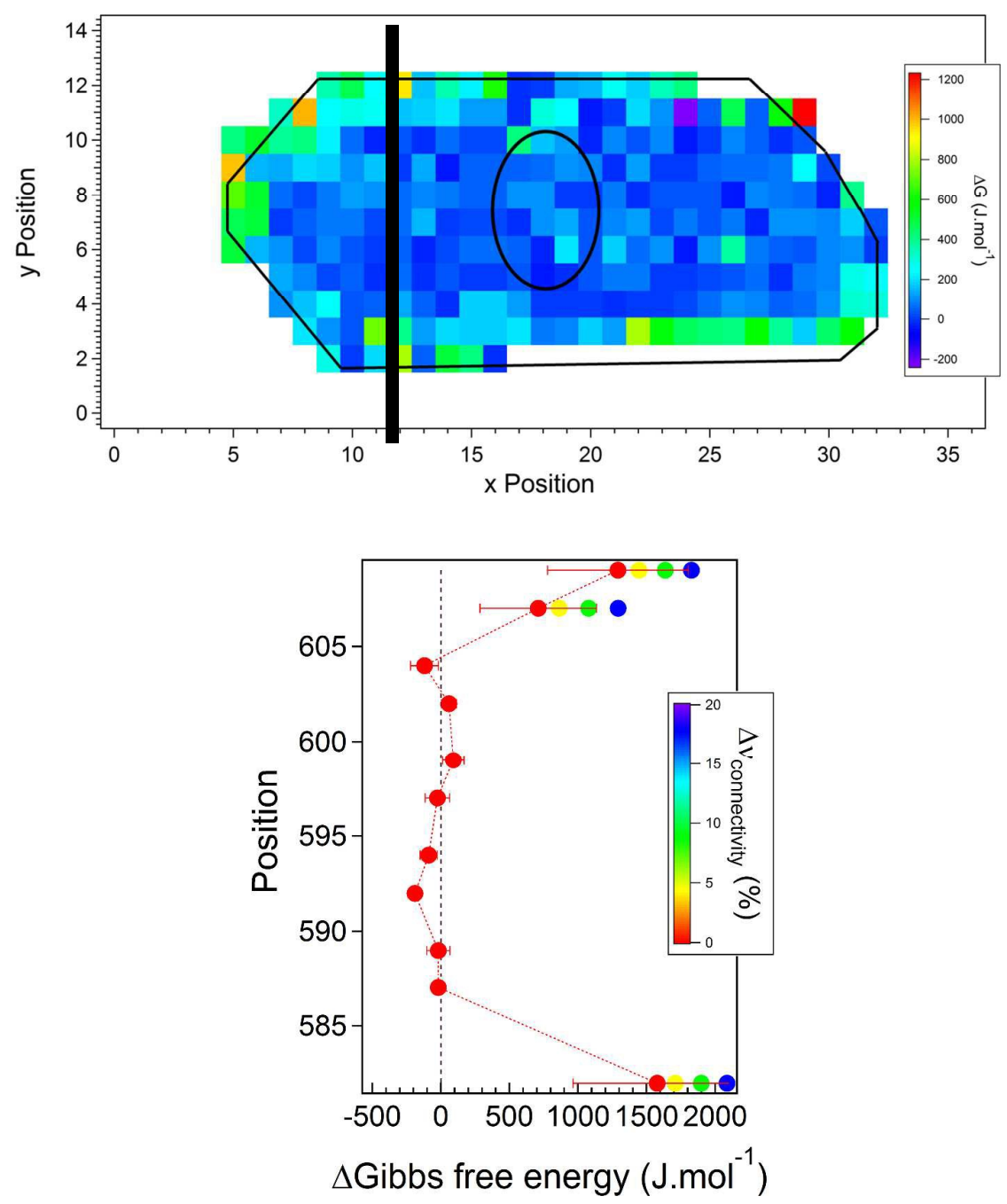

Figure 8. Gibbs free energy mapped as a function of the beam location in the inclusion. Top, over the entire 2D plane. Bottom, along one cross section with a sensitivity analysis (see text). At the center of the inclusion, the mean Gibbs free energy has a bulk-like value (Fig. 8): the mean value is $-6675 \pm 353 \mathrm{~J}_{\mathrm{mol}}{ }^{-1}$ with a root mean square of $78 \mathrm{~J} \cdot \mathrm{mol}^{-1}$. Close to the liquid/solid interfaces, Gibbs free energy significantly increases, reaching very high values: the mean value is $-6457 \pm 945 \mathrm{~J}_{\mathrm{mol}}{ }^{-1}$, with a root mean square of $255 \mathrm{~J} . \mathrm{mol}^{-1}$. The error bars increase as well, while remaining significantly lower than the calculated shift. It should be noted that, contrary to the Raman maps, the IR maps did not record the interfacial signal 
along the entire interfacial area, as already mentioned, due to the Mie scattering and due to the high dependency of the measurement on the beam location and the exact geometry of the interface. The thermodynamic feature arising from the previous cross section is therefore assumed to echo what is really happening inside the Raman-detected layer, even if hidden in the IR map.

Obviously, the calculation of Gibbs free energy assuming constant vibrational modes, except for the intra-molecular stretching, cannot be fully satisfactory. Indeed, Le Caër et al. ${ }^{42}$ have demonstrated that the connectivity band is significantly down-shifted under confinement, while the bending band is almost constant and the librational band is of uncertain but hardly changing behavior. The confinement plays here the role of interfacial effect, as confining has similar spectral features than coming closer to an interface ${ }^{26,42}$. Therefore, we can study the influence of a connectivity trend (if any) on the thermodynamic results in the vicinal area with 5 to $20 \%$ down-shifts (Fig. 8). It appears that the thermodynamic consequence keeps the same trend and even is enforced by taking care of the connectivity. As a result, considering only the intra-molecular stretching certainly under-estimates the real shift.

Eventually, the interfacial effect is thermodynamically characterized by a +0.8 to $+1.4 \mathrm{~kJ} / \mathrm{mol}$ shift in the Gibbs free energy, which makes the vicinal water more reactive than the bulk. This effect is thermodynamically sensitive over relatively long distances, but is steeply attenuated over $1 \mu \mathrm{m}$. Thus, a water layer of significant thickness undergoes this thermodynamic shift and is expected to behave differently than its bulk counterpart. Simple calculations can illustrate this statement, for instance the shift of the phase transition temperature in water (Fig. 9, left) or the solubility with respect to the quartz host (Fig. 9 right). 

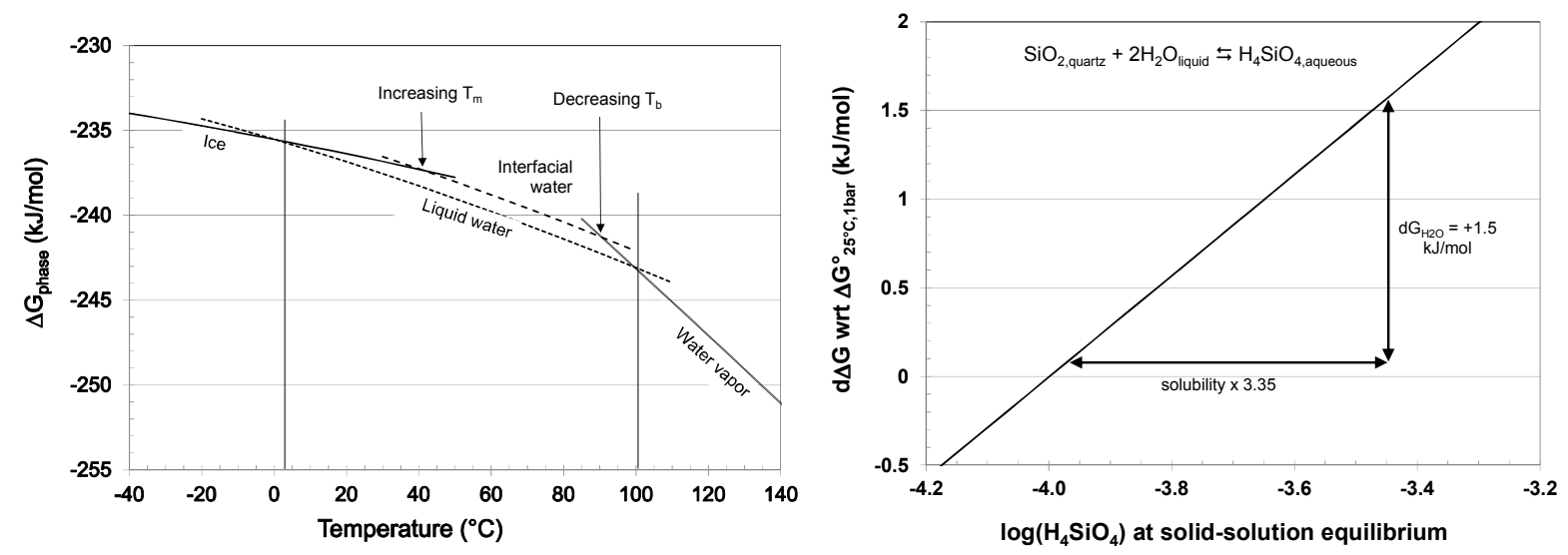

Figure 9. The estimated shifts of the phase transition temperatures (left) and quartz solubility (right) corresponding to the thermodynamic signature of interfacial water presently measured.

It appears that inside the interfacial region, the increasing Gibbs free energy of the liquid causes both a depression of its boiling point, an elevation of its melting point, and enhances the solubility, by a factor of 2.24 for quartz, if the Gibbs free energy of the interfacial water is increased by $1 \mathrm{~kJ} / \mathrm{mol}$ (Fig. 9). The decreasing boiling point is especially interesting, since it is well known that the boiling takes place primarily on the walls which is usually interpreted by the presence of gas pockets trapped in the crevices of the (generally) rough surfaces. However, the SFIs display superheating capabilities which are very contradictory with the presence of such trapped gases. It is why the present calculation may offer a thermodynamic alternative to this classic interpretation: the boiling point is depressed along the wall explaining why this is the preferential locus of bubble appearance, but as long as the inclusion is only filled with liquid (monophasic situation), bubble nucleation must overcome the "normal" (i.e., not crevices-depressed) nucleation barrier, delaying the cavitation.

On the contrary, the higher melting point does not match any experimental or observational records, particularly the well-known persistence of non-freezing layers in capillaries, considered located at the water-silica interfaces ${ }^{45}$. However, these effects are known to be controlled by disjoining pressure effects which are not considered here, and the silica capillary tubes often are not hydrophobic. 
Lastly, the increasing solubility of quartz in interfacial water offers interesting perspectives to re-consider the well-known phenomenon of silica supersaturation in the old groundwaters of large sedimentary basins. Azaroual et al. ${ }^{6}$ have convincingly demonstrated that the Paris basin samples were supersaturated with respect to quartz (Dogger samples) and quartz and chalcedony (Keuper samples). As such old aquifers have reached close-to-equilibrium situation, the authors had to involve a new solid phase in the reasoning, proposing that the Dogger formation hosted invisible nano-sized $(20 \mathrm{~nm})$ quartz, that is to say highly curved quartz in the rock matrix. This proposition is not so self-consistent considering that such curved mineral could have evolved in presence of massive quartz (perfectly visible), through Ostwald ripening processes. In the present line of reasoning, an alternative interpretation can be devised, related to the interface effect on the pore fluids: a rapid calculation of the needed correction amounts to $500 \mathrm{~J} / \mathrm{mol}$ (Fig. 10). This correction is half the value measured on the first micrometer from the inner walls of the fluid inclusions, a satisfactory agreement at this stage.

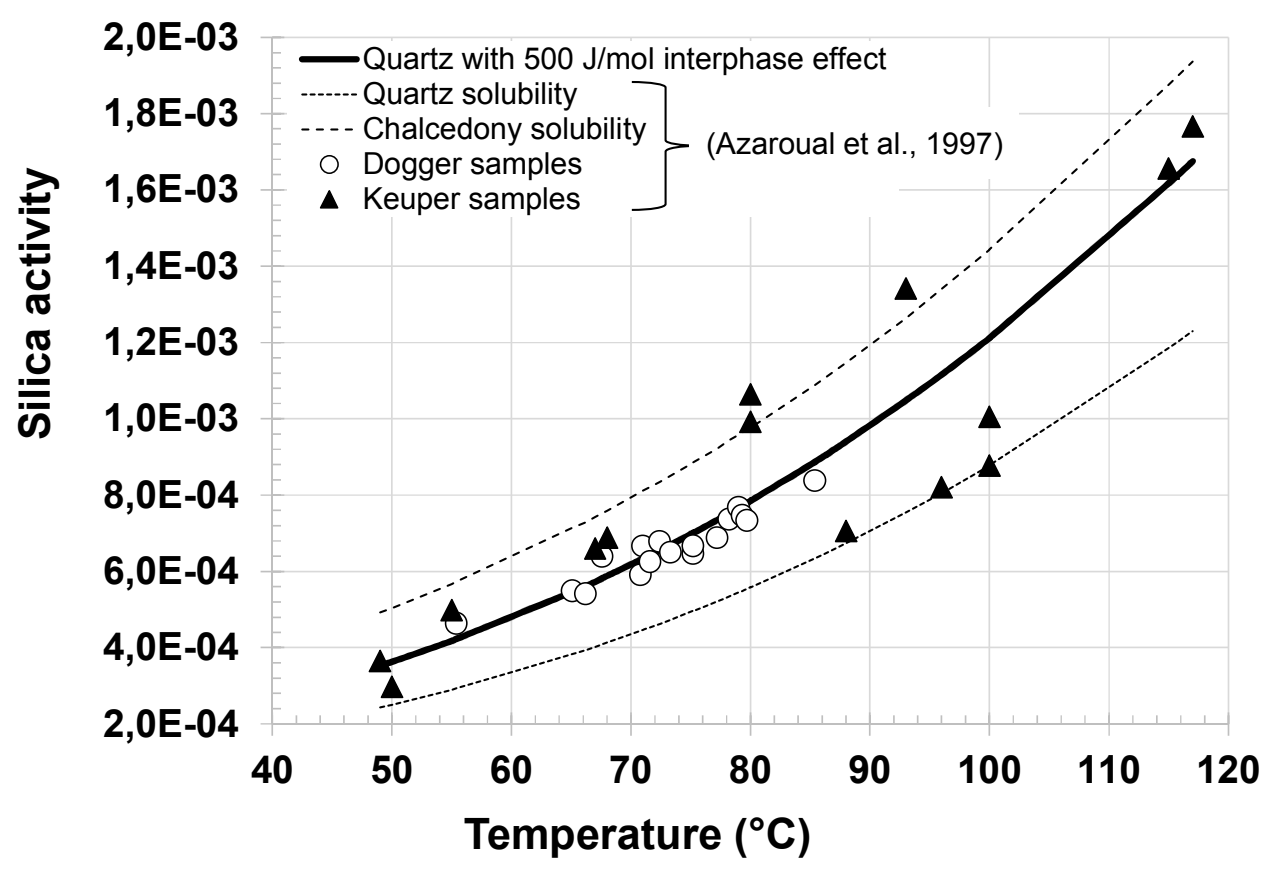


Figure 10. Silica solubility with and without the interfacial effect, as recalculated from Paris Basin datasets (taken from Azaroual et al. ${ }^{6}$ ).

Beyond these first estimates, and according to a classical thermodynamic line of reasoning, such chemical potential modification covering millions of molecules means that there is a specific phase, or a continuous extended interphase domain, standing between bulk solid and bulk solution. In other words, the activity of water is therefore increasing ( $R T \ln a_{\mathrm{w}}$ positive component for $a_{\mathrm{w}}>1$ ) when coming closer to a hydrophobic solid surface. This may sound as a rather counter-intuitive result since hydrophobicity is linked to lack of interactions. At a closer consideration, it appears that the (certainly) large number of dangling $\mathrm{OH}$ bonds gives rise to a large interfacial surface energy ${ }^{30,46}$ which may contribute to the Gibbs free energy of interfacial water.

\section{DISCUSSION}

The present data imply that the thermodynamic properties of liquid water could be modified over micrometer lengths in small cavities as a function of the distance from confining walls. The cause of this noticeable modification is clearly surface-related. It is an equilibrium effect in the thermodynamic sense: the fluid inclusion in quartz, fabricated four years ago, is a closed cavity, and the IR and Raman maps are completely reproducible, even at one year distance.

At this point of our study we may speculate on the specific cause of this mean field effect exhibited within the $\mu \mathrm{m}$ thickness scale. The key feature recorded above is the presence of dangling $\mathrm{OH}$ bonds, signature of poorly water-binding (hydrophobic) inner walls: siloxane bridges are assumed to be responsible for this, most probably formed during the hydrothermal synthesis process $\left(610 \mathrm{MPa}, 458^{\circ} \mathrm{C}\right)$. The density of these $\mathrm{Si}-\mathrm{O}-\mathrm{Si}$ structures certainly defines the weakness of water-solid interactions and drive the H-bonds arrangement in the interfacial 
liquid water. MD simulations, already evoked ${ }^{34-38}$, were able to quantify the degree of hydrophobicity linked to the density of the siloxane groups at the surface. Much like other known processes (hydrophobic solvation), intermolecular forces are assumed to rearrange the H-bonds network to "avoid contact" with the hydrophobic solid (at least in the zones with high enough siloxane density). This finding is quite consistent with a previous observation that hydrophobic surfaces contacting water promote a layer of decreased vapor-like density ${ }^{46}$. The thickness of this vapor-like layer is on the order of 2.5-4 nm, depending on the degree of aeration in water (dissolved gases promote a thicker interfacial layer). In turn, this vapor-like layer may originate a long range "hydrophobic effect", characterized by a strong modification of the intermolecular networking ${ }^{46}$.

This hydrophobic effect, related to the water-solid disconnected (solvophobic) interface, involves primary and secondary hydration forces, known to have characteristic decay lengths of several nanometers and ten of nanometers, respectively ${ }^{47}$. The longer range interactions (1 $\mu \mathrm{m}$ or more), often evoked, involve electrostatics or capillary bridging ${ }^{48}$, which are not supposed to act in the present configuration.

As a result, the only model we found out to reconcile our measurements to the current understanding of the physico-chemistry of interfaces, implies an interfacial thermodynamically oversoluble (because disconnected) liquid-solid interface, associated with an osmotic gradient: a sort of thermos-osmotic effect. First, the weakly connected interface acts locally increasing the thermodynamic water activity, resulting in an interface-driven oversolubility, due to the change in the Gibbs free energy of water (Fig. 9). Consequently, an enhanced concentration of silicic acid is built close to the silica walls. This concentration logically decreases towards the interior of the cavity in the limits of a more or less radial distribution. Accordingly, the in-cavity solution is more concentrated closer to the solid surface than in the centre. A direct outcome of this scheme is that an osmotic gradient would 
be generated from the interface edges to the cavity centre, the second stage of the "model". An osmotic stress develops that is directional and perpendicular to the surface, decreasing with the distance to the interface. The micrometer-thick interphase layer would then be the range over which the osmotic force sufficiently affects the molecular H-bonding network to be recorded with high resolution spectroscopies. Considering that the triggering effect has much to do with surface tension and the status of the water-solid H-bonding connectivity, we could propose that the present finding is a coupled surface-osmotic process.

Interestingly, this proposition can also find support in literature: what is called the "osmotic pressure point of view" of liquid interfaces is exposed in one sub-chapter in the seminal book of Adamson and Gast ${ }^{49}$, treating the surface region as a kind of solution. More recently, Toshev and Platikanov ${ }^{50}$ have introduced a new quantity, the superficial tension of the film in contact with a solid, to account for the long-range interaction forces in wetting films. This would be the surface tension augmented by the interfacial effects evoked above. On the experimental side, the vapor-liquid equilibration studies have abundantly demonstrated ${ }^{51-57}$ that the vapor pressures of pure liquids trapped in sintered stainless steel porous plates are decreasing more than the prediction by the Kelvin equation, for diameters up to $30 \mu \mathrm{m}^{56}$. These anomalous results were interpreted as the effects of long-range forces that promote the formation of special layers along the pores walls with modified surface tension and density, much like the above conclusion.

The attractive side of this hypothesis is that it involves mean field properties that may propagate over relatively long range. Also, it closely associates the solid surface, the solutes distribution and the polar nature of the water solvent. At the same time, it is consistent with the predominance of the lower frequencies in the stretching band of the vibrational spectra over the higher ones. The difficult part is the quantification (and then the validation) of the scope of the effect by combination of these two causes. Also problematic, the driving force to 
generate an incredibly strong mode of dangling $\mathrm{OH}$ bonds is still unclear, even by speculating on a low density, or "nano-bubbles bearing", interfacial region ${ }^{46}$.

\section{CONCLUSION}

Interactions of water with various surfaces in geological, biological, technological environments, often take place in pores, channels, vesicles, membranes, etc., that is to say in confined volumes formed by mineral (inorganic) or organic solid walls. An interfacedependent signal is recorded here with IR spectroscopy in the simplest transmission mode owing to a synchrotron-based collimated beam, supported by a complementary Raman study. The thermodynamic properties deduced from the spectral signature indicate the existence of an interphase domain wherein water may experience a depressed boiling point, an increased melting point, and an oversoluble ability of solids. As a result, an interfacial "interphase water" appears to have a chemical affinity shifted with respect to the bulk water's, which strongly modifies the way confined liquid water interacts with its host solid, on both short and long timescales.

The driving force to build such interphase bears on an interplay of surface effects, linked to the not-H-bonding (hydrophobic) state of the solid-liquid interface, with the osmotic gradient created by the varying silica content from the walls to the centre. According to the present scheme, any hydrophobic (at least partially) solid surface could induce the thick "interphase", as measured here in a special case.

The practical consequences in terms of chemistry in the hydrous world (dominated by water) are obvious and important, strongly modifying our understanding of water-substrate interactions in pores, channels, or any cavities of any sort. Thus, the supersaturation observed in long-lived groundwaters may be driven by the interfacial oversolubility, relayed by the classical nucleation barrier preventing the solid from precipitating in the pore centre. The

pore-size driven solubility ${ }^{7,9-11}$ is also readily accounted for by the growing importance of the 
interfacial area when narrowing the pore, which becomes dominant or exclusive when the pore reaches the micrometer size or less. Interestingly, fields experiments on the "excess air" topic, a "classics" of the groundwater tracing studies, demonstrated that the increased dissolution of gases in soil water was promoted in the fine-grained sediments ${ }^{58}$, for which (in the current line of reasoning) the interfacial thermodynamics is likely to impact the entire pore. As for the laboratory-fields chemical rates discrepancies, the modified interfacial reactivity should impact the reactive surface area side of the debate. The role of film reactivity ${ }^{12}$, the presence of coatings (through their hydrophobic-hydrophilic features) at the water-solid interfaces ${ }^{44}$, the thermodynamic signature of interfaces versus the extension of surface area ${ }^{59}$, are all interpretations of the discrepancies which could naturally integrate the interfacial oversolubility behavior. This finding also has interesting extensions to geodynamics through the wet/dry materials rheology ${ }^{60-61}$ : it is known for long that the presence of water leads to a strong mechanical weakening of the deforming rock matrix.

Eventually, any scientific area related to the stability and evolution of the chemical properties in water-containing cavities ${ }^{62}$, or interested to use water as a green solvent, may benefit from these results.

Acknowledgments. This work has received support from Region Centre, grant 201100070577 SIRE, and from the French Agency for Research (Agence Nationale de la Recherche, ANR) through the grant CONGE BLAN-610-01. It also benefited from the beamtime allocations n²0130094 and n²0130776 from SOLEIL synchrotron. The supports from Equipex Planex ANR-11-EQPX-36 and labex Voltaire ANR-10-LABX-100-01 are also acknowledged. The measurements at ID19 were done by E. Boller who also was of great help to interpret the data, in collaboration with O. Rozenbaum. We are indebted to four referees who greatly contributed to improve the quality of the initial manuscript. 


\section{REFERENCES}

[1] G.E. Brown, V.E. Henrich, W.H. Casey, D.L. Clark, C. Eggleston, A. Felmy, D.W.

Goodman, M. Grätzel, G. Maciel, M.I. McCarthy, K.H. Nealson, D.A. Sverjensky, M.F. Toney and J.M. Zachara, Chem. Rev., 1999, 99(1), 77-174.

[2] M.A. Henderson, Surf. Sci. Rep., 2002, 46, 1-308.

[3] L.J. Michot, F. Villiéras, M. François, I. Bihannic, M. Pelletier and J.-M Cases, C. R. Geoscience, 2002, 334, 611-631.

[4] P. Fenter and N.C. Struchio, Prog. Surf. Sci., 2004, 77, 171-258.

[5] H.J.G.E. Gardeniers, Anal. Bioanal. Chem., 2009, 394, 385-397.

[6] M. Azaroual, C.Fouillac, and J.M. Matray, Chem. Geol., 1997, 140, 167-179.

[7] Y. Tardy in Petrology of laterites and tropical soils. Taylor \& Francis, 1997, 408 p.

[8] C.I. Steefel and P.C. Lichtner, J. Hydrol., 1998, 209, 200- 224.

[9] A. Putnis, A. and G. Mauthe, Geofluids, 2001, 1, 37-41.

[10] S. Emmanuel and B. Berkowitz, Geophys. Res. Letters, 2007, 34, art.L06404.

[11] T. Yokoyama, S. Nakashima, T. Murakami, L. Mercury and Y. Kirino, Appl. Geochem., 2011, 26, 1524-1534 (2011).

[12] N. Nishiyama and T. Yokoyama, Geochim. Cosmochim. Acta, 2013, 122, 153-169.

[13] T. Tsukuhara, A. Hibara, Y. Ikeda and T. Kitamori, Angew. Chem. Int. Ed., 2007, 46, $1180-1183$.

[14] P. Mentré in L'eau dans la cellule. Masson, 1995, 292 p.

[15] Z. Liu, K. Muldrew, R.G. Wan. and J.A.W. Elliott, Phys. Rev. E, 2003, 67, art.061602.

[16] S. Le Caër, G. Klein, D. Ortiz, M. Lima, S. Devineau, S. Pin, J.-B. Brubach, P. Roy, S. Pommeret, W. Leibl, R. Righini and J.P. Renault, Phys. Chem. Chem. Phys., 2014, 16, 22841. 
[17] I. Bergonzi, L. Mercury, J.-B. Brubach and P. Roy, Phys. Chem. Chem. Phys., 2014, 16, 24830-2484.

[18] K.I. Shmulovich and C.M. Graham, Contrib. Mineral. Petrol., 2004, 146, 450-462.

[19] K.I. Shmulovich, L. Mercury, R. Thiéry, C. Ramboz and M. El Mekki, Geochim. Cosmochim. Acta., 2009, 73, 2457-2470.

[20] K.I. Shmulovich, B.W.D. Yardley and C.M. Graham, Geofluids, 2006, 6, 157-167.

[21] F. Jamme, B. Lagarde, A. Giuliani, G.A. Garcia and L. Mercury, J. Phys.: conf. series, 2013, 425, 142002.

[22] Y. Maréchal, in The hydrogen bond and the water molecule: the physics and chemistry of water, aqueous, and bio media. Elsevier, Amsterdam, The Netherlands, 2007.

[23] V. Mazet, C. Carteret, D. Brie, J. Idier and B. Humbert, Chemom. Intell. Lab. Syst., 2005, 76(2), 121-133.

[24] J.-B. Brubach, A. Mermet, A. Filabozzi, A. Gershel, and P. Roy, J. Chem. Phys., 2005, 105, $430-435$.

[25] C. Boissière, J.-B. Brubach, A. Mermet, G. de Marzi, C. Bourgaux, E. Prouzet, and P.Roy, J. Phys. Chem. B, 2002, 106, 132-135.7

[26] L. Mercury, F. Jamme and P. Dumas, Phys. Chem. Chem. Phys., 2012, 14, 2864-2874.

[27] P. Bassan, H.J. Byrne, F. Bonnier, J. Lee, P. Dumas and P. Gardner, Analyst, 2009, 134, 1586-1593.

[28] X. Wei and Y.R. Shen, Phys. Rev. Lett., 2001, 86, 4799.

[29] Q. Du, E. Freysz and Y.R Shen, Science, 1994a, 264, 826.

[30] Q. Du, E. Freysz and Y.R. Shen, Phys. Rev. Lett., 1994b, 72, 238.

[31] Q. Du, R. Superfine, E. Freysz and Y.R. Shen, Phys. Rev. Lett., 1993, 70, 2313.

[32] L. F. Scatena, M.G. Brown, and G.L. Richmond, Science, 2001, 292, 908-912.

[33] Y.R. Shen, and V. Ostroverkhov, Chem. Rev., 2006, 106, 1140-1154. 
[34] R. Zangi, J. Phys.: Cond. Mat., 2004, 16, S5371-S5388.

[35] J.W. Wang, A.G. Kalinichev and R.J. Kirkpatrick, J. Phys. Chem. B, 2005, 109, 1430814313.

[36] J. Wang, A.G. Kalinichev and R.J. Kirkpatrick, J. Phys. Chem. C, 2009, 113, $11077-$ 11085.

[37] R. Vacha, R. Zangi, J.B.F.N. Engberts and P. Jungwirth, J. Phys. Chem. C, 2008, 112, 7689-7692.

[38] B. Rotenberg, A.J. Patel and D. Chandler, J. Am. Chem. Soc., 2011, 133, 20521-20527.

[39] G.M. Rignanese, J.C. Charlier and X. Gonze, Phys. Chem. Chem. Phys., 2004, 6, 19201925.

[40] J. Yang and E.G.Wang, Curr. Opinion Solid State Materials Sci., 2006, 10, 33-39.

[41] G. Onori and A. Santucci, J. Phys. Chem., 1993, 97, 5430-5434.

[42] S. Le Caër, S. Pin, S. Esnouf, Q. Raffy, J.P. Renault, J.-B. Brubach, G. Greff and P. Roy, Phys. Chem. Chem. Phys., 2011, 13, 17658-17666.

[43] S.H. Lee and P. J. Rossky, J. Chem. Phys., 1994, 100, 3334-3345.

[44] M.R. Lee, D.J. Brown, M.E. Hodson, M. Mackenzie and C.L. Smith, Mineral. Mag., 2008, 72(6), 1319-1328.

[45] N.V. Churaev, V.D. Sobolev and V.M. Starov, J.Colloid Interf. Sci., 2002, 247, 80-83

[46] D.A. Doshi, E.B. Watkins, J.N. Israelachvili, and J. Majewski, Proc. Natl. Acad. Soc. USA, 2005, 102(27), 9458-9462.

[47] B.W. Ninham, T.T. Duignan and D.F. Parsons, Curr. Opinion Colloid \& Interf. Sc., 2011, 16, 612-617.

[48] M.U. Hammer, T.H. Anderson, A. Chaimovich, M.S. Shell and J. Israelachvili, Faraday Discuss., 2010, 146, 299-308. 
[49] A.W. Adamson. and A. P.Gast in Physical chemistry of surfaces. J. Wiley \& Sons, New York, $6^{\text {th }}$ ed., 1997, 808p.

[50] B. Toshev and D. Platikanov, Colloids and Surfaces A: Physicochem. Eng. Aspects, 2006, 291, 177-180.

[51] J.L. Shereshefsky, J. Am. Chem. Soc., 1928, 50, 2966-2980.

[52] D.H. Everett and W.I. Whitton, Proc. Royal Soc. London A, 1955, 230, 91-110.

[53] N.L. Coleburn and J.L. Shereshefsky, J. Colloid Interf. Sci., 1972, 38, 84-90.7

[54] G.C. Yeh, M.S. Shah and B.V. Yeh, Langmuir, 1986, 2, 90-96.

[55] G.C. Yeh, B.V. Yeh, S.T. Schmidt, M.S. Yeh, A.M. McCarthy and W.J. Celenza, Desalination, 1991, 81, 161-187.

[56] F.A. Abu Al-Rub and R. Datta, Fluid Phase Equilib., 1998, 147, 65-83.

[57] F. A. Abu Al-Rub and R. Datta, Fluid Phase Equilib., 1999, 162, 83-96.

[58] S. Klump, Y. Tomonaga, P. Kienzler, W. Kinzelbach, T. Baumann, D.M. Imboden and R. Kipfer, Geochim. Cosmochim. Acta, 2007, 71, 1385-1397.

[59] E.H. Oelkers, P.A. Bjorkum, O. Walderhaug, P.H. Nadeau and W.M. Murphy Applied Geochem., 2000, 15, 295-309.

[60] E. Burov, Mar. Petrol. Geol., 2011, 28, 1402-1443.

[61] P. Cordier and J.C. Doukhan, Eur. J. Mineral., 1989, 1, 221-237.

[62] L. Mercury, K.I. Shmulovich, I. Bergonzi, A.Canizares and P. Simon J. Phys. Chem. C (accepted), doi: 10.1021/acs.jpcc.6b01700. 